\title{
ART for HIV in India
}

\author{
TamizharasiS ${ }^{1^{*}}$, Ramamoorthi $\mathrm{M}^{2}$, Chellapan $\mathrm{N}^{3}$, Induhelen. $\mathrm{R}^{4}$ \\ ${ }^{1}$ Professor, Department of Pharmaceutics, Nandha College of Pharmacy, Erode, \\ Tamilnadu, Email:Thamilarasi.mmc@gmail.com \\ ${ }^{2}$ Associate Professor, Department of Pharmacology, Nandha College of Pharmacy, Erode, \\ Tamilnadu, Email: ramamoorthi@nandhapharmacy.org \\ ${ }^{3}$ Assistant Professor, Department of Pharmaceutics, Nandha College of Pharmacy, Erode, \\ Tamilnadu, Email: chellapan@nandhapharmacy.org \\ ${ }^{4}$ Professor, Department of Community Health Nursing, Nandha College of Nursing, Erode, \\ Tamilnadu, Email:induhelen@gmail.com \\ ${ }^{*}$ Corresponding Author
}

\begin{abstract}
Antiretroviral therapy (ART) is the treatment for HIV/AIDS through medicine which decrease the viral load in the patient. It is suggested for everyone who has HIV to reduce the spread of infection in the body. This paper gives the clear idea about the medicine used in ART treatment in India. However these medicine will only reduce the viral infection and manage the chronic condition.
\end{abstract}

Keywords: HIV, ART, AIDS, viral load.

\section{Introduction}

HIV stands for human immunodeficiency virus. These virus affects the human immune system function and make the body less resistant against infection. The virus invades the CD4 cells which is important for the white blood cells. This makes the body less immune to $\mathrm{HIV}$ and also to other related infection.

When HIV is not treated on time it eventually develop into AIDS. Not all people having HIV will get AIDS but when not treated properly they develop the condition to get the disease.to control the infection and to reduce the viral load different medicine combination are given. The medicine should have a good adherence to the body to reduce the viral load. ART was introduced in early 1960 for treating AIDS. Prior monotherapy was followed. As the virus is short lived it makes the replication faster so there a need for faster move of medicine in the body. Slowly the monotherapy was replaced by ART.

The treatment of HIV involves different kinds of ARVs. Each type has its own way to attack the virus and reduce the spread in the body. The most common use are three types: Nucleoside reverse transcriptase inhibitors (NRTIs), Non-nucleoside reverse transcriptase inhibitors (NNRTIs), Protease inhibitors (PIs). There is another type called Integrase inhibitors which works by blocking the enzyme integrase.

These treatment proved that there is increased rate of survival rate and lowers the chance of getting AIDS. However these medicine does not cure HIV but helps to reduce the risk and helps to maintain good immune system against infection. The medicine prevents the virus from multiplying in faster rate. Even though they control the spread of infection each type has its side effect which shows different symptoms in the patient body. The common side effects are nausea, vomiting, fatigue, diarrhea and other chronic problems.

Additionally strict diet and regular exercise should be followed along with the medicine to control the infection. The patient are requested to take the medicine regularly in specific time and in specific intervals. Many researches are made to improve the adherence of the medicine in the body. However the patient should take the medicine properly to reduce the viral load. If they are not taking properly the virus which are sensitive will be killed but the virus organism which are more resistant to drug will live and replicate to increase the viral load. So it is important to take the medicine properly without interruption. There needs to be at least two active drugs from multiple drug classes in an antiretroviral regimen in order for it to be successful.

\section{Nucleoside reverse transcriptase inhibitors (NRTIs)}

These work by blocking the enzyme called reverse transcriptase. The reverse transcriptase are responsible for converting the viral RNA to call DNA which replicate and destroys the cell own self. The 
NRTls works by by blocking RT before HIV genetic material combines with the genetic code of an infected cell, we disrupt the copying of HIV genetic material by RT. It Mimic the building blocks used by RT to copy HIV genetic material, so that HIV genetic material does not get copied. CD4 cells, or T cells, are part of the immune system, and HIV normally enters these cells. Once HIV enters CD4 cells, it begins copying itself. To do so, the virus must convert its genetic material, RNA, into DNA. In reverse transcription, an enzyme called reverse transcriptase is required. NRTIs prevent the virus' reverse transcriptase from accurately converting RNA into DNA. Without DNA, HIV cannot make copies of itself. The Food and Drug Administration (FDA) has approved seven NRTIs for HIV treatment. These medications are available both as single medicines and in combination. The formulations include: zidovudine (Retrovir) ,lamivudine (Epivir), abacavir sulfate (Ziagen), didanosine (Videx), delayed-release didanosine (Videx EC), stavudine (Zerit),emtricitabine (Emtriva),enofovir disoproxil fumarate (Viread), lamivudine and zidovudine (Combivir), abacavir and lamivudine (Epzicom),abacavir, zidovudine, and lamivudine (Trizivir), tenofovir disoproxil fumarate and emtricitabine (Truvada), tenofovir alafenamide and emtricitabine (Descovy). The NRTIs come in pill form that is taken by mouth. NRTI treatment is usually followed by two NRTIs plus one drug from another class of antiretroviral drugs. Test results, which provide important details about a person's condition, are used by healthcare providers to choose treatment. If the person has taken antiretroviral drugs in the past, it is also considered by healthcare providers when choosing treatment. This is the main method for overseeing instances of HIV. The accompanying tips can assist with guaranteeing adherence to treatment: Take the prescription simultaneously every day,Utilize a week after week pill box that has compartments for every day of the week. These containers are accessible in many drug stores, Join taking the medicine with an undertaking that is played out each day. This makes it part of the day by day schedule. Utilize a schedule to mark off the days when drug was taken, Set a caution update for taking the drug on a telephone or PC,Download a free application that can give updates when it's an ideal opportunity to take the drug, A quest for "update applications" will give numerous choices. The following are a couple to attempt,Ask a relative or companion to give updates for take the drug, Orchestrate to get message or telephone informing updates from the medical services supplier.

NRTIs can cause secondary effects. Some secondary effects are more normal than others, and these medications can influence various individuals in an unexpected way. Every individual's response depends to some degree on which medicates their medical care supplier recommends and what different medications that individual takes. By and large, fresher NRTIs, for example, tenofovir, emtricitabine, lamivudine, and abacavir, cause less aftereffects than more established NRTIs, like didanosine, stavudine, and zidovudine.

Albeit these secondary effects aren't normal, it's vital to realize that they can happen and to examine them with a medical care supplier. Some incidental effects can be kept away from or controlled.Any individual who encounters these extreme aftereffects should contact their medical services supplier promptly to decide if they should continue to take the prescription. They shouldn't quit taking the medication all alone.

Managing secondary effects can be terrible, however halting the medicine might permit the infection to foster opposition. This implies that the drug might quit filling in too to keep the infection from repeating. The medical services supplier might have the option to change the blend of medications to diminish aftereffects. The danger of aftereffects might be higher relying upon an individual's clinical history and way of life. As indicated by the $\mathrm{NIH}$, the danger of some antagonistic aftereffects might be higher if the individual l: is female or large (the main danger that is higher is for lactic acidosis), consumes different medications has other ailments, Likewise, liquor abuse can build the danger of liver harm. An individual who has any of these danger elements should converse with their medical services supplier prior to taking NRTIs.

NRTIs are a portion of the drugs that have made HIV the board conceivable. For these significant medications, fresher forms cause less serious aftereffects than past renditions, however some incidental effects might in any case happen for any of these drugs.It's significant for individuals whose medical care suppliers have recommended NRTIs to adhere to their therapy intend to oversee HIV. Assuming that they have aftereffects from antiretroviral treatment, they can attempt these ways to diminish those incidental effects. All the more significantly, they can converse with their medical care supplier, who can make ideas or change their therapy intend to assist with calming incidental effects.

Non-nucleoside reverse transcriptase inhibitors (NNRTIs)

NNRTIs (Non-nucleoside Reverse Transcriptase Inhibitors) block RT before HIV genetic code interacts with the inherent code of the contaminated cell, preventing it from operating. NNRTIs have a low inherent 
to deterrence, as well as the extra damage of needing twice-consistent dose (associated to treatment adherence). Elvitegravir, which is more potent, can be taken once a day with cobicistat (an inhibitor of elvitegravir processing) and two NRTIs. Because of the increased risk of block-related disappointments, NNRTIs are generally not advised as part of second-line regimens.

HIV-1 and HIV-2 are the two types of HIV, and both can cause serious illness (AIDS). The HIV type 1 (HIV-1) strain is responsible for the majority of AIDS-related pollutions. Between HIV-1 and HIV-2, there are significant differences that provide insights into infection progression, tropism, and pathogenesis. HIV2 , in particular, is less quickly transmitted and, in general, less harmful than HIV-1.

The Food and Drug Administration now recommends five NNRTIs (nevirapine, NVP; delavirdine, DLV; efavirenz, EFV; etravirine, ETR; and rilpivirine, RPV) (FDA). Furthermore, with the exception of DLV, all of them have received assistance from the European Union. EFV (Atripla) and RPV (Complera/Eviplera) have been combined with two NRTIs (emtricitabine and tenofovir) as a single pill, once-daily regimen to improve adherence and reduce the risk of medication errors. When the professionals connected with the coplan recommend Atripla and Complera/Eviplera for the treatment of HIV-1 infection, they are the prescriptions of choice.

Drugs with a minimal genetic barrier that only require a single alteration to confer resistance are known as unique NNRTIs, and cross-deterrent impacting these NNRTIs is common. Second-age NNRTIs, when all factors are considered, are compounds with a greater hereditary block to deterrence. Despite the fact that hepatotoxicity and an exaggerated rash are linked, they are all typically safe and suffering. EFV, on the other hand, creates central tangible framework (CNS) ultimate results when using NVP. The vast majority of them have a lengthy plasmatic half-life and are administered in a step-by-step fashion.

The cutting-edge period of NNRTIs is currently undergoing clinical fresh research. The need for new helpful specialists who can assist people overcome obstacles and flourish prompted the formation of new NNRTIs. Development against wild-type HIV-1 and therapeutically suitable oddity strains improves these mixes. Treatment discontent appears to be dynamically rare with contemporary antiretroviral blends comprising advanced NNRTIs, making it harder for the new NNRTls to demonstrate their commonness with yielding over the first- and second-age NNRTIs.

NNRTIs with a low hereditary barrier, such as NVP, DLV, and EFV, require just a single mutation to confer resistance, and cross-checking is common. Second- generation NNRTIs, such as ETR and RPV, have a greater inherited deterrence. The FDA has backed the two ages, as has the European Union, with the exception of DLV. The clinical advancement of particular NNRTIs is now unstoppable, and these drugs have been dubbed "state of the art NNRTIs." These combinations, as second-generation NNRTIs, have the benefit of being medicines that work against both HIV1 wild-type and clinically important oddity strains.

\section{Protease Inhibitors (PIs)}

Blocks a protease chemical that breaks lengthy protein strands into minimum functional proteins and synthetics that are predicted to form a mature illness. It halts the development of new well-known particles. Because of strong plasma protein limiting, which makes Pls concentration lower in refuge places like the genital plot, most Pls are simply adequately swallowed in the gastrointestinal plot and their dispersal anyway real block is by and large modest. PIs have a strong prosperity profile when it comes to foreboding situations. Gl solicitation and outright dissociation of the entrails are the most commonly reported transient uncomfortable experiences. Lipodystrophy is a disease characterised by long-term receptivity to PIs, particularly the early mixes. Metabolic agitation effects (hyperglycemia and hyperlipidemia) are common, regardless of morphological abnormalities (fat rot and fat proclamation). In 1995, saquinavir (Invirase and Fortovase) was approved, followed by the launch of ritonavir (Norvir) and indinavir (Crixivan) in 1996, nelfinavir (Viracept) in 1997, and amprenavir (Agenerase and Prozei) in 1999. To the degree that PIs have advanced in HAART treatment, these five specialists discuss the earliest and, by and large, the most widely used Pls at the end of the 1990s. Saquinavir is a peptidomimetic HIV protease inhibitor that is inexplicably confounded by its nearly full firstpass digestion by cytochrome P450. . Saquinavir is typically well tolerated, with mild gastrointestinal symptoms being the most often seen adverse effects. Ritonavir is a protease inhibitor that has been a source of controversy in HIV treatment. Oral osmosis is quite high and is unaffected by meals. Indinavir is a manufactured peptidomimetic ruthless inhibitor of the HIV aspartyl protease, and its major drawback is that it does not have an inductive effect on the hepatic CYP impetuses, which affects its own digesting. Nelfinavir is an inhibitor of HIV protease, the enzyme responsible for HIV propeptide post-translational therapy. A distinct variation in protease quality acts as a deterrent.

Diverse protease impediment alterations were detected when treatment disenchantment with a single PI occurred, regularly accomplishing wide class 
hindrance. Second-generation techniques to combat PI resistance improvement were developed in the 2000s, including lopinavir (Aluviran and Koletra) in 2000 and fosamprenavir (Lexiva and Telzir) and atazanavir (Reyataz) in 2003. Its viability, tolerability, and virological impact were all addressed. Lopinavir is the most widely used and long-acting PI. The drug was specifically designed to counteract the flaws of previous specialists in this field. Along with lipid abnormalities, the event of gastrointestinal disruptive affects is genuinely hostile.

The advantages of PIs are that they save NNRTI for a while, they have a higher genetic limit to obstruction, and they have extraordinary resilience to disillusionment. Metabolic disarrays, gastrointestinal side effects, liver toxicity, CYP3A4 inhibitors and substrates, possibility for medication interactions, PR stretch prolongation are all examples of PIs obstructions. Food and a low stomach $\mathrm{pH}$ affect absorption.

\section{HIV medicine interference}

Some HIV/AIDS medications prevent HIV from infecting CD4 invulnerable structure cells, such as combination inhibitors, which prevent HIV from infecting cellphones. Different particles on CD4 cells are blocked by CCR5 antagonists and post-association inhibitors. HIV must attach to two types of particles on a cell's surface in order to sully it. HIV is prevented from entering telephones by blocking both of these iotas.

Association inhibitors attach to a particular protein on HIV's outer surface and prevent it from spreading. This prevents HIV from infecting the phone. People sometimes use many medications: Pharmacokinetic enhancers contribute to the sufficiency of some HIV/AIDS treatments. The degradation of the other medicine is slowed by a pharmacokinetic enhancer. This permits the treatment to stay in the body for longer and at a greater concentration. Multidrug mixes combine two or more HIV/AIDS medications.

\section{Conclusion}

It's critical to take your medicines on a regular basis, as directed by your medical care provider. If you skip doses or don't stick to a strict schedule, your treatment may fail, and your HIV infection may grow resistant to the medications.

Antiretroviral medications can have unintended consequences. The majority of these side effects are tolerable, however a few of them are not. Any side effects you're experiencing should be reported to your medical care provider. Don't stop taking your prescription without first speaking with your pharmacist. The individual could be able to advise you on the most effective way to deal with the consequences. Your medicine may be changed by your provider at any time. Antiretroviral medications aren't just used to treat HIV. They are taken by certain

people to help them from contracting HIV. PrEP (preopenness prophylaxis) is a treatment for those who do not currently have HIV but are at a high risk of contracting it. PEP (post-openness prophylaxis) is a treatment for those who have been exposed to HIV.

\section{Reference}

1. Santos, L.O.; Marinho, F.A.; Altoé, E.F.; Vitório, B.S.; Alves, C.R.; Britto, C.; Motta, M.C.; Branquinha, M.H.; Santos, A.L.; d'Avila-Levy, C.M. HIV aspartyl peptidase inhibitors interfere with cellular proliferation, ultrastructure and macrophage infection of Leishmania amazonensis. PLOS One, 2009, 3, e4918.

2. Desjeux, P., Alvar, J. Leishmania/HIV co-infections: epidemiology in Europe. Ann. Trop. Parasitol., 2003, 97, 3-15.

3. Savoia, D.; Allice, T.; Tovo, P.A. Antileishmanial activity of HIV protease inhibitors. Internat. J. Antimicrobial. Agents. ,2005, 26, 92-4.

4. Robertson, C.D. The Leishmania mexicana proteasome. Mol. Biochem. Parasitol., 1999, 103, 49-60.

5. Trudei, N.; Garg, R.; Messier, N.; Sundar, S.; Ouellette, M.; Tremblay, M.J. Intracellular survival of Leishmania species that cause visceral leishmaniasis is significantly reduces by HIV-1 protease inhibitors. J. Infect. Dis., 2008, 198, 1292-9.

6. Yao, C.; Donelson, J.E.; Wilson, M.E. The major surface protease (MSP or GP63) of Leishmania sp. Biosynthesis, regulation of expression, and function. Mol. Biochem. Parasitol., 2003, 132, 1-16. Mottram, J.C.; Coombs, G.H.; Alexander, J. Cysteine peptidases as virulence factors of Leishmania. Curr. Opin. Microbiol., 2004, 7, 375-81.

7. Hill, D.; Dubey, J.P. Toxoplasma gondii: transmission, diagnosis and prevention. Clin. Microbiol. Infect., 2002, 8, 634-40.

8. Montoya, J.; Liesenfled, O. Toxoplasmosis. Lancet., 2004, 363, 1965-76.

9. Luft, B.; Chua, A. Central Nervous System toxoplasmosis in HIV: pathogenesis, diagnosis and therapy. Curr. Infect. Dis. Rep., 2000, 2, 358-62.

10. Cates, W.; J. Estimates of the incidence and prevalence of sexually transmitted diseases in the United States. Sex. Transm., 1999, 26, S2-7.

11. Wolner-Hanssen, P.; Krieger, J.N; Stevens, C.E; Kiviat, N.B.; Koutsky, L.; Critchlow , C.; DeRouen, T.; Hillier, S.; Holmes, K. Clinical manifestations of vaginal trichomoniasis. JAMA., 1989, 261, 571-6.

12. Farthing, M.J. Giardiasis. Gastroenterol. Clin. North. Am., 1996, 25, 493-515 